\title{
THE SPECTRAL MATRICES OF TODA SOLITONS AND THE FUNDAMENTAL SOLUTION OF SOME DISCRETE HEAT EQUATIONS
}

\author{
LUC HAINE
}

\begin{abstract}
The Stieltjes spectral matrix measure of the doubly infinite Jacobi matrix associated with a Toda $g$-soliton is computed, using Sato theory. The result is used to give an explicit expansion of the fundamental solution of some discrete heat equations, in a series of Jackson's $q$-Bessel functions. For Askey-Wilson type solitons [?, ?], this expansion reduces to a finite sum. This generalizes a result in [?], which corresponds to the limit $q \rightarrow 1$.
\end{abstract}

To Pierre van Moerbeke for his sixtieth birthday

\section{INTRODUCTION}

The fundamental solution $K(y \mid x, t)$ of the heat equation

$$
u_{t}=u_{x x}-V(x) u, \quad u(x, 0)=\delta(x-y),
$$

has been linked with soliton theory from the early days, by providing a tool for obtaining the integrals of the motion of the Korteweg-de Vries $(\mathrm{KdV})$ equation, see [?, ?]. In recent years, the problem has been turned around, by using soliton theory as a tool to understand the fundamental solution, for some special classes of potentials $V(x)$.

Motivated by the work of Berest and Veselov [?] on Hadamard's problem for the wave equation and its relation with the bispectral problem (see [?] and references therein), Grünbaum [?, ?] suggested that a similar connection might be present in the context of the heat equation. In [?], this was confirmed for the first few examples of the family of potentials $V_{i}(x)=2\left(\log \tau_{i}(x)\right)^{\prime \prime}$, with $\tau_{0}=1, \tau_{1}=x, \tau_{2}=$ $x^{3} / 3-s_{3}, \ldots$ formed by the rational solutions of the $\mathrm{KdV}$ equation, which provide the so-called rank 1 solutions of the bispectral problem as originally formulated in [?]. For these potentials, the "Hadamard coefficients" $H_{k}(x, y)$ in the asymptotic expansion (valid in general for

Date: September 12, 2004.

2000 Mathematics Subject Classification. 35Q51, 37K20, 39A13.

Key words and phrases. Heat kernel expansions, Toda lattice hierarchy. 
$t \downarrow 0$ and for $x$ close to $y$ ) of the fundamental solution of (??)

$$
K(y \mid x, t) \sim \frac{e^{-\frac{(x-y)^{2}}{4 t}}}{2 \sqrt{\pi t}}\left(1+\sum_{k=1}^{\infty} H_{k}(x, y) t^{k}\right),
$$

vanish for $k \geq i+1$. Thus, in these cases, the expansion gives rise to an exact formula consisting of a finite number of terms.

A similar "finite number of terms phenomenon" was observed in [?] and established by Grünbaum and Iliev in [?], when the space variable $x$ in (??) is discrete, that is for an equation of the type

$$
u_{t}(n, t)=L u(n, t), \quad n \in \mathbb{Z},
$$

with $L$ a doubly infinite tridiagonal matrix. If $L$ belongs to the family of rational solutions of the Toda lattice hierarchy, the fundamental solution $K(m \mid n, t)$ of (??) with initial condition $u(n, 0)=\delta_{n m}$, can be expressed as a finite sum of terms of the form $e^{-2 t} t^{r} I_{k}(2 t), r \geq 0$, with $I_{k}(t)$ the Bessel functions of imaginary argument. The rational solutions of the Toda lattice hierarchy were constructed in [?, ?], as part of a study of the rank 1 solutions to a discrete-continuous version of the bispectral problem, linking the problem with rational curves with only cusp-like singularities as in the pioneering work of Wilson [?]. The proof in [?] is based on an orthogonality relation satisfied by the wave function (in the sense of Sato theory), that was established in full generality in [?], using singular algebraic curve theory.

In this paper, I propose to further explore the "finite number of terms phenomenon" found in [?, ?, ?] by studying the case when $L$ in (??) is a doubly infinite Jacobi matrix, leading (when taken as initial condition) to a soliton solution of the Toda lattice equations [?, ?]. For sake of brevity, I shall call these Jacobi matrices "Toda solitons". It is well known (see for instance [?]) that the fundamental solution of the heat equation (??) can be written in terms of the eigenfunctions and eigenvalues of the Schrödinger operator $L=-\partial^{2} / \partial x^{2}+V(x)$. In the case of solitons, there is a continuous spectrum and a finite number of eigenvalues (bound states). For "Toda solitons", we shall establish the following formula for the fundamental solution of (??)

$$
\begin{array}{r}
K(m \mid n, t)=\frac{1}{2 \pi i} \oint e^{t \frac{\left(z+z^{-1}\right)}{2}} \phi(n, z) \phi\left(m, z^{-1}\right) \frac{\mathrm{d} z}{z} \\
+\sum_{j=1}^{g} c_{j}^{2} e^{t \frac{\left(z_{j}+z_{j}^{-1}\right)}{2}} \phi\left(n, z_{j}\right) \phi\left(m, z_{j}\right) .
\end{array}
$$

In this formula, the integral is taken over the unit circle in the complex $z$ plane; $\phi(n, z)$ is the discrete Jost function that satisfies the asymptotic 
condition $\phi(n, z) \sim z^{n}$ as $n \rightarrow+\infty$ on $\left.|z|=1 ; z_{j} \in\right]-1,1[, j=1, \ldots, g$ denote the bound states of $L$, i.e. $L \phi\left(n, z_{j}\right)=\left(z_{j}+z_{j}^{-1}\right) / 2 \phi\left(n, z_{j}\right)$, and $c_{j}$ are the norming constants. I refer the reader to Section 3 , for a quick review of discrete inverse scattering theory.

The main issue in (??) is to show that when $t=0, K(m \mid n, 0)=\delta_{n m}$. In Sections 2 and 3, we give a proof of these orthogonality relations, in the spirit of [?], using Sato theory applied to some special irreducible rational singular curves with double points. For second-order formally self-adjoint difference operators on the integers, the analogue of the classical Weyl-Titchmarsh-Kodaira theory for second-order differential operators on the line, can be found in [?]. Instead of a spectral measure which is characteristic of the semi-infinite case (both continuous and discrete), we have now a two by two spectral matrix measure. In the so-called limit point case, to which the operators we are dealing with belong to, the spectral matrix is unique. In Section 3, the orthogonality relations are interpreted as providing the Stieltjes spectral matrix measure of a "Toda soliton", thus linking our curve theoretic approach to the orthogonality relations with classical spectral theory.

The situation considered in [?] corresponds to the limiting case in (??) where all the bound states accumulate at $z_{j}= \pm 1$. In the case of pure solitons, with all $z_{j}$ 's belonging to ] - 1, 1[ and distinct, (??) can be expanded in a convergent series of Bessel functions $I_{k}(t)$ of imaginary argument, but this series never leads to a finite sum formula (see Remark 5.3). In Section 5, we replace in (??) the exponential factors by the product of $q$-exponentials $e_{q}(t z / 2) e_{q}\left(t z^{-1} / 2\right)$, which is the generating function for the Jackson's $q$-Bessel functions $I_{k}(t ; q)$ (of imaginary argument), see [?, ?, ?]. Again, $K(m \mid n, t)$ can be expanded in a series of the functions $I_{k}(t ; q)$ (convergent for $t$ small enough), see Theorem 5.2. However, if we pick now the bound states to be

$$
z_{1}= \pm q^{\frac{r_{1}}{2}}, \ldots, z_{g}= \pm q^{\frac{r_{g}}{2}}, \quad 0<q<1,
$$

with $r_{1}, \ldots, r_{g}$ arbitrary positive integers (with an arbitrary choice of the \pm signs, keeping the $z_{j}$ 's distinct), this expansion reduces to a finite sum, using an apparently new identity satisfied by the functions $I_{k}(t ; q)$, which is established in Section 4 of the paper. With the modification of the exponential by the $q$-exponential, (??) becomes the fundamental solution of a purely discrete heat equation

$$
D_{q, t} u(n, t)=\left(L+\frac{(q-1) t}{4} I d\right) u(n, t), \quad n \in \mathbb{Z},
$$

(with a time-dependent discrete Schrödinger operator), where $D_{q, t}$ denotes the $q$-derivative with respect to $t$. Remarkably, the condition (??) 
precisely defines the so-called Askey-Wilson type solitons [?, ?], that provide the rank 1 solutions of a difference - $q$-difference version of the bispectral problem, that was initiated in [?] in the aim of generalizing the celebrated Askey-Wilson polynomials [?], see also [?].

\section{TODA SOLITONIC CURVES}

We consider a special case of a procedure in algebraic geometry [?, ?] that enables one to construct rank 1 commutative rings of difference operators, starting with an irreducible affine algebraic curve (eventually singular), with two non-singular marked points $P_{\infty}$ and $Q_{\infty}$ at infinity. The presentation below was worked out in [?], though some complements will be added.

A Toda solitonic curve is an irreducible rational singular curve with double points, given by the equation

$$
C: \quad v^{2}=\left(u^{2}-4\right) \prod_{j=1}^{g}\left(u-\left(z_{j}+z_{j}^{-1}\right)\right)^{2}
$$

where $z_{1}, \ldots, z_{g}$ are distinct non-zero complex numbers inside the unit circle, $\left|z_{j}\right|<1$. It is rationally parametrized by putting

$$
\begin{aligned}
& u=z+z^{-1}, \\
& v=\left(z-z^{-1}\right) \prod_{j=1}^{g}\left(\left(z+z^{-1}\right)-\left(z_{j}+z_{j}^{-1}\right)\right),
\end{aligned}
$$

$z \in \mathbb{P}^{1}(\mathbb{C})$. In this parametrization, the two marked points $P_{\infty}$ and $Q_{\infty}$ correspond respectiveley to $z=\infty$ and $z=0$.

We write $s=\left(s_{1}, s_{2}, \ldots\right)$ and $\exp (s, z)=\exp \left(\sum_{k=1}^{\infty} s_{k} z^{k}\right)$. Fixing a collection of $g$ non-zero complex numbers $\varepsilon_{1}, \ldots, \varepsilon_{g}$, the Baker-Akhiezer function (called the wave function in the context of Sato theory, see $[?])$, is defined to be the unique function of the form

$$
w(n, s, z)=\exp (s, z) z^{n}\left\{1+\sum_{j=1}^{g} \frac{w_{j}(n, s)}{z-z_{j}^{-1}}\right\},
$$

subject to the $g$ conditions

$$
\operatorname{res}_{z=z_{j}^{-1}} w(n, s, z)-\varepsilon_{j} w\left(n, s, z_{j}\right)=0 .
$$

The dual Baker-Akhiezer function (also called the dual wave function), is defined by

$$
w^{*}(n, s, z)=\exp ^{-1}(s, z) z^{-n}\left\{1+\sum_{j=1}^{g} \frac{w_{j}^{*}(n, s)}{z-z_{j}}\right\},
$$


and is determined by the $g$ conditions

$$
\operatorname{res}_{z=z_{j}} w^{*}(n, s, z)+\varepsilon_{j} w^{*}\left(n, s, z_{j}^{-1}\right)=0 .
$$

The wave and dual wave functions satisfy an identity that will be important for us.

Proposition 2.1. (Bilinear identities).

$$
\operatorname{res}_{z=\infty} w(n, s, z) w^{*}\left(m, s^{\prime}, z\right)=0, \quad \forall n \geq m \text { and } \forall s, s^{\prime} .
$$

Proof. It follows immediately from (??) and (??) that

$$
\left\{\operatorname{res}_{z=z_{j}^{-1}}+\operatorname{res}_{z=z_{j}}\right\} w(n, s, z) w^{*}\left(m, s^{\prime}, z\right)=0, \quad \forall n, m \in \mathbb{Z} .
$$

When $n \geq m$, we see from (??) and (??) that $w(n, s, z) w^{*}\left(m, s^{\prime}, z\right)$ is holomorphic at $z=0$. Thus, by the residue theorem, we deduce that

$$
\begin{aligned}
\operatorname{res}_{z=} & w(n, s, z) w^{*}\left(m, s^{\prime}, z\right)= \\
& -\sum_{j=1}^{g}\left\{\operatorname{res}_{z=z_{j}^{-1}}+\operatorname{res}_{z=z_{j}}\right\} w(n, s, z) w^{*}\left(m, s^{\prime}, z\right)=0, \forall n \geq m,
\end{aligned}
$$

which establishes the proposition.

The next proposition introduces the tau-function, in the sense of Sato theory.

Proposition 2.2. The tau-function corresponding to the wave function in (??) is

$$
\tau_{n}(s)=\operatorname{det}\left(z_{j}^{-(i-1)}-\mu_{j}(s) z_{j}^{2 n+i-1}\right)_{1 \leq i, j \leq g},
$$

with

$$
\mu_{j}(s)=\mu_{j} \exp \left(\sum_{k=1}^{\infty} s_{k}\left(z_{j}^{k}-z_{j}^{-k}\right)\right)
$$

and

$$
\mu_{j}=\varepsilon_{j} \frac{\prod_{k=1, k \neq j}^{g}\left(z_{j}^{-1}-z_{k}^{-1}\right)}{\prod_{k=1}^{g}\left(z_{j}-z_{k}^{-1}\right)}=\varepsilon_{j} z_{j}^{2-g} \frac{\prod_{k=1, k \neq j}^{g}\left(z_{k}-z_{j}\right)}{\prod_{k=1}^{g}\left(z_{j} z_{k}-1\right)} .
$$

Proof. It is easy to check that the unique function of the form (??), satisfying (??), is given by

$$
\begin{aligned}
w(n, s, z)=\frac{\exp (s, z)}{\prod_{j=1}^{g}\left(z-z_{j}^{-1}\right)} & \\
& \times \frac{\operatorname{det}\left(z_{j}^{-(i-1)}-\mu_{j}(s) z_{j}^{2 n+i-1} ; z^{n+i-1}\right)}{\operatorname{det}\left(z_{j}^{-(i-1)}-\mu_{j}(s) z_{j}^{2 n+i-1}\right)_{1 \leq i, j \leq g}},
\end{aligned}
$$


with $\mu_{j}(s)$ as in (??), (??); the notation in the numerator means the determinant of the $(g+1) \times(g+1)$ matrix with $(i, j)$ entries as indicated there for $1 \leq i \leq g+1,1 \leq j \leq g$, and entries $z^{n+i-1}, 1 \leq i \leq g+1$, in the last column.

Introducing the notation

$$
s-\left[z^{-1}\right]=\left(s_{1}-\frac{1}{z}, s_{2}-\frac{1}{2 z^{2}}, s_{3}-\frac{1}{3 z^{3}}, \ldots\right),
$$

one computes easily from (??) that

$$
\mu_{j}\left(s-\left[z^{-1}\right]\right)=\frac{z-z_{j}}{z-z_{j}^{-1}} \mu_{j}(s) .
$$

Then, elementary row manipulations with the determinant in the numerator of (??) lead to

$$
w(n, s, z)=\exp (s, z) z^{n} \frac{\tau_{n}\left(s-\left[z^{-1}\right]\right)}{\tau_{n}(s)},
$$

which is the equation defining the tau-function (up to a non-zero constant) in Sato theory. This finishes the proof.

It is a well known fact [?, ?] that the Baker-Akhiezer function $w(n, s, z)$ is the common eigenfunction of a rank 1 commutative ring of difference operators. More precisely, for any meromorphic function $f$ on $C$, which is holomorphic on $C \backslash\left\{P_{\infty}, Q_{\infty}\right\}$, there is a finite difference operator $L_{f}$ such that $L_{f} w(n, s, z)=f(z) w(n, s, z)$. The size of the operator $L_{f}$ is determined by the order of the poles of $f$ at $P_{\infty}$ and $Q_{\infty}$. Namely, if $f$ has a pole of order $i$ at $P_{\infty}$ and a pole of order $j$ at $Q_{\infty}$, then the operator $L_{f}$, thought of as a finite band matrix, has $i$ diagonals above the main diagonal and $j$ diagonals below it.

For the curve (??), the function $u=z+z^{-1}$ has a simple pole at $P_{\infty}$ and $Q_{\infty}$, hence there exists a unique tridiagonal operator $L_{u}$, satisfying $L_{u} w(n, s, z)=\left(z+z^{-1}\right) w(n, s, z)$. By a standard technique (see [?, ?]), one can show that this three-term recurrence relation satified by the wave function, is explicitly given by

$$
\begin{array}{r}
\frac{\tau_{n-1}(s) \tau_{n+1}(s)}{\tau_{n}(s)^{2}} w(n-1, s, z)+\frac{\partial}{\partial s_{1}} \log \frac{\tau_{n+1}(s)}{\tau_{n}(s)} w(n, s, z)+w(n+1, s, z) \\
=\left(z+z^{-1}\right) w(n, s, z)
\end{array}
$$

We conclude this section with two properties which are special to Toda solitonic curves, that will play a crucial role in the paper. 
Proposition 2.3. The wave and the dual wave function of a Toda solitonic curve are related by the following equation

$$
\exp \left(s, z^{-1}\right) w^{*}(n, s, z)=\frac{\tau_{n-1}(s)}{\tau_{n}(s)} z^{-1} \exp ^{-1}(s, z) w\left(n-1, s, z^{-1}\right) .
$$

Proof. An easy check shows that the only function of the form (??) satisfying the $g$ conditions in (??) is given by

$$
\begin{aligned}
w^{*}(n, s, z)=(-1)^{g} & \frac{\exp ^{-1}(s, z)}{\prod_{j=1}^{g}\left(z-z_{j}\right)} \\
& \times \frac{\operatorname{det}\left(z_{j}^{-(i-2)}-\mu_{j}(s) z_{j}^{2 n+i-2} ; z^{g+1-n-i}\right)}{\operatorname{det}\left(z_{j}^{-(i-1)}-\mu_{j}(s) z_{j}^{2 n+i-1}\right)_{1 \leq i, j \leq g}},
\end{aligned}
$$

where the determinant in the numerator denotes the determinant of a $(g+1) \times(g+1)$ matrix, using the same conventions as in (??). A straightforward computation using (??), (??) and (??), gives the relation (??), which establishes the proposition.

Proposition 2.4. Put

$$
\varepsilon_{j}=c_{j}^{2} z_{j} \Leftrightarrow \mu_{j}=c_{j}^{2} z_{j}^{3-g} \frac{\prod_{k \neq j, k=1}^{g}\left(z_{k}-z_{j}\right)}{\prod_{k=1}^{g}\left(z_{j} z_{k}-1\right)} .
$$

Then, the $\tau$-function (??) can be expressed (up to an inessential factor) in Flaschka's form

$$
\tau_{n}(s)=\operatorname{det}\left(z_{j}^{-(i-1)}\right)_{1 \leq i, j \leq g} \operatorname{det} B(n, s),
$$

with $B(n, s)$ the $g \times g$ matrix with entries

$$
B(n, s)_{i j}=\delta_{i j}+c_{i}(s) c_{j}(s) \frac{\left(z_{i} z_{j}\right)^{n+1}}{1-z_{i} z_{j}},
$$

and

$$
c_{j}(s)=c_{j} \exp \left(\sum_{k=1}^{\infty} s_{k}\left(z_{j}^{k}-z_{j}^{-k}\right) / 2\right) .
$$

Proof. Expanding the determinant (??) as a polynomial in the $\mu_{j}(s)$, using the Vandermonde determinant formula, we find

$$
\begin{aligned}
\tau_{n}(s)=\operatorname{det}\left(z_{j}^{-(i-1)}\right)_{1 \leq i, j \leq g} & \sum_{J \subseteq\{1, \ldots, g\}}(-1)^{\sharp J} \prod_{j \in J} \mu_{j}(s) z_{j}^{2 n} \\
\times & \prod_{\substack{j, k \in J \\
j<k}} \frac{z_{j}-z_{k}}{z_{j}^{-1}-z_{k}^{-1}} \prod_{j \in J, k \notin J} \frac{z_{j}-z_{k}^{-1}}{z_{j}^{-1}-z_{k}^{-1}} .
\end{aligned}
$$


By substituting (??) in (??), using (??) and (??), we find after some computations that

$$
\begin{aligned}
\tau_{n}(s)=\operatorname{det}\left(z_{j}^{-(i-1)}\right)_{1 \leq i, j \leq g} & \\
& \times \sum_{J \subseteq\{1, \ldots, g\}} \prod_{j \in J} \frac{c_{j}^{2}(s) z_{j}^{2(n+1)}}{1-z_{j}^{2}} \prod_{\substack{j, k \in J \\
j<k}}\left(\frac{z_{j}-z_{k}}{1-z_{j} z_{k}}\right)^{2} .
\end{aligned}
$$

We now show that the sum in (??) agrees with $\operatorname{det} B(n, s)$ in (??). To this end, we use an argument in [?] (pp. 365-366), noticing that $B(n, s)=I d+D C D$, with $I d$ the identity matrix, $D=\operatorname{diag}\left(c_{1}(s) z_{1}^{n+1}, \ldots, c_{g}(s) z_{g}^{n+1}\right)$ a diagonal matrix, and $C$ the $g \times g$ matrix $C=\left[\left(1-z_{i} z_{j}\right)^{-1}\right]_{1 \leq i, j \leq g}$. Hence, the determinant of $B(n, s)$ boils down to the sum of all the principal minors of the matrix $D C D$. It is not difficult to evaluate these minors by means of the Cauchy determinant formula

$$
\operatorname{det}\left(\frac{1}{1-x_{j} y_{k}}\right)_{1 \leq j, k \leq N}=\frac{\prod_{1 \leq j<k \leq N}\left(x_{j}-x_{k}\right)\left(y_{j}-y_{k}\right)}{\prod_{1 \leq j, k \leq N}\left(1-x_{j} y_{k}\right)},
$$

which, setting $x_{j}=y_{j}=z_{j}$, leads to the sum in (??). This concludes the proof.

\section{TODA SOLITONS AND THEIR SPECTRAL MATRICES}

We start this section by relating the various constructions in Section 2 to the data used in the discrete version of the inverse scattering problem. We follow [?] (see also [?]), where the theory summarized below can be found. One considers in the Hilbert space $l^{2}$ of squaresummable, doubly infinite complex sequences $\{f(n) ;-\infty<n<\infty\}$, the following eigenvalue problem

$$
(L f)(n) \equiv a_{n-1} f(n-1)+b_{n} f(n)+a_{n} f(n+1)=\frac{z+z^{-1}}{2} f(n),
$$

where $z$ denotes a (complex) spectral parameter. Here $a_{n}>0$ for all $n \in \mathbb{Z}$; it is assumed that $a_{n} \rightarrow 1 / 2$ and $b_{n} \rightarrow 0$ rapidly (exponentially, for example) as $|n| \rightarrow \infty$, see [?] for the precise assumptions.

The discrete Jost functions are the solutions $\phi(n, z)$ and $\psi(n, z)$ of $L f=\frac{z+z^{-1}}{2} f$, satisfying the asymptotic conditions

$$
\begin{aligned}
\left|\phi(n, z)-z^{n}\right| \rightarrow 0, & n \rightarrow+\infty, \\
\left|\psi(n, z)-z^{-n}\right| & \rightarrow 0, \quad n \rightarrow-\infty,
\end{aligned}
$$

for $|z|=1$. Still restricting to $|z|=1$, one can show that

$$
\psi(n, z)=\alpha(z) \phi\left(n, z^{-1}\right)+\beta(z) \phi(n, z),
$$


with $|\alpha(z)|^{2}-|\beta(z)|^{2}=1$. The function $S(n, z)=\psi(n, z) / \alpha(z)$ has the asymptotic behaviour

$$
S(n, z)=z^{-n}+\frac{\beta(z)}{\alpha(z)} z^{n}, \quad n \rightarrow+\infty .
$$

It is called the scattering solution; $R(z)=\beta(z) / \alpha(z)$ is called the reflection coefficient and $1 / \alpha(z)$ is the transmission coefficient. The eigenvalues of $L$ are simple, finite in number, and correspond to real values $\left.z_{j} \in\right]-1,1\left[, j=1, \ldots, g\right.$. If $\lambda_{j}=\left(z_{j}+z_{j}^{-1}\right) / 2$ is an eigenvalue, and if $\xi\left(n, z_{j}\right)$ denotes the corresponding eigenfunction normalized by $\sum_{n=-\infty}^{\infty} \xi\left(n, z_{j}\right)^{2}=1$, then

$$
\xi\left(n, z_{j}\right) \sim c_{j} z_{j}^{n}, \quad n \rightarrow+\infty,
$$

for certain $c_{j}>0$, called the norming constants. The quantities $(R(z)$, $\left.z_{1}, \ldots, z_{g}, c_{1}, \ldots c_{g}\right)$, constitute the collection of scattering data from which the Jacobi operator $L$ can be recovered. We shall (somewhat loosely) refer to the $z_{j}$ 's, rather than the $\lambda_{j}$ 's, as the discrete spectrum.

In the so-called reflectionless case $R(z)=0$, and the operator $L$ is reconstructed via the following formulas

$$
a_{n}=\frac{1}{2} \frac{\sqrt{\tau_{n-1}(0) \tau_{n+1}(0)}}{\tau_{n}(0)}, \quad b_{n}=\left.\frac{1}{2} \frac{\partial}{\partial s_{1}} \ln \frac{\tau_{n}(s)}{\tau_{n-1}(s)}\right|_{s=0},
$$

where $\tau_{n}(s)$ is defined as in (??), (??) and (??), and the $c_{j}$ 's and $z_{j}{ }^{\prime}$ 's in these formulas coincide with the norming constants and the eigenvalues as described above. Notice that, after deleting the irrelevant first factor, formula (??) for $\tau_{n}(s)$ makes it clear that $\tau_{n}(s)>0$.

Definition 3.1. The doubly infinite Jacobi matrix $L(? ?)$ with entries defined as in (??), will be called a Toda g-soliton.

The reason for this terminology is that the solution of the Toda lattice equations

$$
\dot{L}\left(s_{1}\right)=\left[L_{+}\left(s_{1}\right)-L_{-}\left(s_{1}\right), L\left(s_{1}\right)\right],
$$

with initial condition $L(0)=L, L$ as in (??), is a g-soliton solution of these equations; here $L_{+}$(resp. $L_{-}$) denote the strictly upper (resp. lower) part of $L$. When flowing, the operator $L\left(s_{1}\right)$ stays reflectionless, with the same eigenvalues $\lambda_{j}=\left(z_{j}+z_{j}^{-1}\right) / 2$ and norming constants $c_{j}\left(s_{1}\right)=c_{j} \exp \left(s_{1}\left(z_{j}-z_{j}^{-1}\right) / 2\right)$, see [?]. When the $c_{j}$ 's are allowed to flow according to (??), one gets a solution of the full Toda lattice hierarchy. In the rest of the paper, we shall not be interested in the evolution of $L$ along the flows of the Toda lattice hierarchy, and we shall always put $s=(0,0, \ldots)$ in our formulas. For sake of brevity, we shall write $\tau_{n}$ instead of $\tau_{n}(0)$. 
Lemma 3.2. In the reflectionless case, the discrete Jost function $\phi(n, z)$ defined in (??), is related as follows to the wave function $w(n, 0, z)$

$$
\phi(n, z)=\sqrt{\frac{\tau_{n-1}}{\tau_{n}}} z w(n-1,0, z)
$$

with $\tau_{n}=\tau_{n}(0)$.

Proof. Via the transformation

$$
f(n)=\sqrt{\frac{\tau_{n-1}}{\tau_{n}}} g(n),
$$

using (??), the three-term recurrence relation (??) passes over into

$$
\frac{\tau_{n-2} \tau_{n}}{\tau_{n-1}^{2}} g(n-1)+\left.\frac{\partial}{\partial s_{1}} \ln \frac{\tau_{n}(s)}{\tau_{n-1}(s)}\right|_{s=0} g(n)+g(n+1)=\left(z+z^{-1}\right) g(n) \text {. }
$$

Comparing this recurrence with (??), shows that it is satisfied by taking $g(n)=w(n-1,0, z)$. Thus, the function on the right-hand side of (??) satisfies (??) with $a_{n}$ and $b_{n}$ as in (??). To conclude that this function agrees with the Jost function $\phi(n, z)$, it suffices to check that the asymptotic condition (??) is satisfied for $|z|=1$. This is easily done from (??), remembering that $\left|z_{j}\right|<1$, using the Vandermonde determinant formula and the fact that $\lim _{n \rightarrow+\infty} \tau_{n}$ is a constant independent of $n$, see (??). The lemma is established.

Theorem 3.3. Let $\phi(n, z)$ be the discrete Jost function given by (??). The following orthogonality relations hold

$$
\frac{1}{2 \pi i} \oint \phi(n, z) \phi\left(m, z^{-1}\right) \frac{\mathrm{d} z}{z}+\sum_{j=1}^{g} c_{j}^{2} \phi\left(n, z_{j}\right) \phi\left(m, z_{j}\right)=\delta_{n m},
$$

for all $m, n \in \mathbb{Z}$, with $\oint$ denoting the integral over the unit circle in the complex z plane.

Proof. Using (??) and (??) (with $s=0$ ), (??) is equivalent to

$$
\begin{aligned}
& \frac{1}{2 \pi i} \oint w(n-1,0, z) w^{*}(m, 0, z) \mathrm{d} z+ \\
& \frac{\tau_{m-1}}{\tau_{m}} \sum_{j=1}^{g} c_{j}^{2} z_{j}^{2} w\left(n-1,0, z_{j}\right) w\left(m-1,0, z_{j}\right)=\delta_{n m}, \forall m, n \in \mathbb{Z} .
\end{aligned}
$$

We evaluate the integral in (??) by residues. We note from (??) and (??), that the integrand has poles inside the unit circle only at $z=0$ and $z=z_{j}, j=1, \ldots, g$. 
Using (??), we have

$\operatorname{res}_{z=z_{j}} w(n-1,0, z) w^{*}(m, 0, z)=-\varepsilon_{j} w\left(n-1,0, z_{j}\right) w^{*}\left(m, 0, z_{j}^{-1}\right)$.

Substituting (??) in this last equation, and expressing $w^{*}\left(m, 0, z_{j}^{-1}\right)$ in terms of $w\left(m-1,0, z_{j}\right)$ using (??) with $s=0$ and $z=z_{j}^{-1}$, gives

$$
\begin{aligned}
\operatorname{res}_{z=z_{j}} w(n-1,0, z) & w^{*}(m, 0, z) \\
& =-\frac{\tau_{m-1}}{\tau_{m}} c_{j}^{2} z_{j}^{2} w\left(n-1,0, z_{j}\right) w\left(m-1,0, z_{j}\right) .
\end{aligned}
$$

From (??) we conclude that, in order to establish (??), it remains to show that

$$
\operatorname{res}_{z=0} w(n-1,0, z) w^{*}(m, 0, z)=\delta_{n m}, \quad \forall m, n \in \mathbb{Z} .
$$

From (??), (??) and (??) (all with $s=0$ ), we obtain the following expansions around $z=0$

$$
w(n, 0, z)=z^{n}\left(\frac{\tau_{n+1}}{\tau_{n}}+O(z)\right) \text { and } w^{*}(n, 0, z)=z^{-n}\left(\frac{\tau_{n-1}}{\tau_{n}}+O(z)\right) .
$$

Thus

$$
w(n-1,0, z) w^{*}(m, 0, z)=z^{n-m-1}\left(\frac{\tau_{n} \tau_{m-1}}{\tau_{n-1} \tau_{m}}+O(z)\right), \text { around } z=0,
$$

which establishes (??) for $n \geq m$. On the other hand, from (??) (with $s=0$ ), we deduce that

$$
\begin{aligned}
& \operatorname{res}_{z=0} w(n-1,0, z) w^{*}(m, 0, z)=-\frac{\tau_{n} \tau_{m-1}}{\tau_{n-1} \tau_{m}} \\
& \times \operatorname{res}_{z=\infty} w(m-1,0, z) w^{*}(n, 0, z) .
\end{aligned}
$$

By the bilinear identities (??) with $s=s^{\prime}=0$, the right-hand side of (??) is zero for $m-1 \geq n$, thus establishing (??) for $n \leq m-1$. This concludes the proof of the theorem.

As announced in the Introduction, we end this section by giving the spectral interpretation of (??). Since we are dealing with bounded doubly infinite Jacobi matrices, we are automatically in the so-called limit point case at $\pm \infty$, for which the spectral matrix is unique (up to a change of basis of fundamental solutions). The general form of the orthogonality relations is as follows, see [?] and [?] for an application linked with a doubly infinite version of the Jacobi polynomials. Given $\phi(n, z)$ and $\psi(n, z)$ a fundamental system of solutions for $L f=\frac{z+z^{-1}}{2} f$ on $|z|=1$, let us define for $n$ a non-negative integer, the matrix

$$
P_{n}(z)=\left(\begin{array}{cc}
\phi(-n-1, z) & \psi(-n-1, z) \\
\phi(n, z) & \psi(n, z)
\end{array}\right), \quad n \geq 0 .
$$


Then, there exists a unique Stieltjes spectral matrix measure $\mathrm{d} \Sigma(z)$, such that the following orthogonality relations hold

$$
\oint P_{n}(z) \mathrm{d} \Sigma(z) P_{m}(z)^{T}=\delta_{n m} I d, \forall n, m \geq 0,
$$

where $\oint$ denotes the integral over the unit circle in the complex $z$ plane, $P_{m}(z)^{T}$ is the transpose of $P_{m}(z)$ and $I d$ is the identity matrix. The next corollary gives the spectral interpretation of Theorem 3.3.

Corollary 3.4. In the basis of the discrete Jost functions $\phi(n, z)$ and $\psi(n, z)$ as defined in (??) and (??), the Stieltjes spectral matrix measure of a Toda g-soliton is given by

$$
\mathrm{d} \Sigma(z)=\left(\begin{array}{cc}
0 & 1 / 2 \\
1 / 2 & 0
\end{array}\right) \frac{1}{\alpha(z)} \frac{\mathrm{d} z}{2 \pi i z}+\sum_{j=1}^{g} c_{j}^{2}\left(\begin{array}{cc}
1 & 0 \\
0 & 0
\end{array}\right) \delta\left(z-z_{j}\right)
$$

with $z_{1}, \ldots, z_{g}$, the discrete spectrum, $c_{1}, \ldots, c_{g}$, the norming constants, $\delta(z)$ Dirac's delta function and

$$
\frac{1}{\alpha(z)}=\prod_{j=1}^{g} \frac{z_{j} z-1}{z-z_{j}}
$$

the transmission coefficient.

Proof. With $\mathrm{d} \Sigma(z)$ defined as in (??), a straightforward computation shows that the orthogonality relations (??) amount to

$$
\begin{aligned}
\frac{1}{4 \pi i} \oint\{\phi(n, z) & \psi(m, z)+\psi(n, z) \phi(m, z)\} \frac{\mathrm{d} z}{\alpha(z) z} \\
& +\sum_{j=1}^{g} c_{j}^{2} \phi\left(n, z_{j}\right) \phi\left(m, z_{j}\right)=\delta_{n m}, \forall n, m \in \mathbb{Z} .
\end{aligned}
$$

In the reflectionless case, we have that $\beta(z)=0$ in (??). Thus, we can replace in (??) $\psi(n, z) / \alpha(z)$ by $\phi\left(n, z^{-1}\right)$, showing that these orthogonality relations precisely amount to those we established in (??). It is well known (see for instance [?]) that for a Toda $g$-soliton, the transmission coefficient is given by (??). This proves the corollary.

Remark 3.5. To the best of my knowledge, formulas (??) and (??) have not appeared in the literature on the Toda lattice. However, related issues have been considered for semi-infinite Jacobi matrices in [?], and for the inverse scattering on the line in [?]. It seems to me interesting that the curve theoretic approach to the orthogonality relations applies when the curves have worse singularities than double points, which leads to nonselfadjoint eigenvalue problems, see [?]. 
4. Q-Bessel FUnCTIONS With IMAGINARY ARGUMENT

Let $0<q<1$. We denote by

$$
e_{q}(z)=\sum_{n=0}^{\infty} \frac{(1-q)^{n} z^{n}}{(q ; q)_{n}}=\frac{1}{((1-q) z ; q)_{\infty}}, \quad|z|<\frac{1}{1-q},
$$

the $q$-analogue of the exponential function $e^{z}$, where the $q$-shifted factorials are defined by

$$
\begin{gathered}
(a ; q)_{n}=\prod_{j=0}^{n-1}\left(1-a q^{j}\right), n=1,2, \ldots ; \quad(a ; q)_{0}=1 ; \\
(a ; q)_{\infty}=\prod_{j=0}^{\infty}\left(1-a q^{j}\right) .
\end{gathered}
$$

It follows immediately from (??) and (??) that

$$
e_{q}\left(q^{r} z\right)=((1-q) z ; q)_{r} e_{q}(z), r=1,2, \ldots
$$

We introduce a $q$-analogue of the Bessel functions with imaginary argument, via the generating function

$$
e_{q}\left(\frac{t z}{2}\right) e_{q}\left(\frac{t z^{-1}}{2}\right)=\sum_{k=-\infty}^{\infty} I_{k}(t ; q) z^{k}
$$

for $\frac{(1-q)|t|}{2}<|z|<\frac{2}{(1-q)|t|}$. These functions relate to one of the two $q$-analogues of the Bessel functions introduced by Jackson [?]. The two (closely related) Jackson's $q$-analogues of Bessel functions were revived in [?], and are denoted by $J_{k}^{(1)}(z ; q)$ and $J_{k}^{(2)}(z ; q)$ (see also [?], pp. 25-26). With these notations, $I_{k}(t ; q)=(-i)^{k} J_{k}^{(1)}(i(1-q) t ; q)$.

In the next section, we shall need the following identity.

Proposition 4.1. Let $r=1,2,3, \ldots$ Then, for any $k \in \mathbb{Z}$, one has

$$
\begin{aligned}
& \left(q^{\frac{k r}{2}}-q^{-\frac{k r}{2}}\right) I_{k}(t ; q)= \\
& \sum_{j=1}^{r}\left[\begin{array}{l}
r \\
j
\end{array}\right]_{q} q^{\frac{j(j-r-1)}{2}}\left(\frac{q-1}{2}\right)^{j} t^{j}\left(I_{k-j}\left(q^{-r / 2} t ; q\right)-I_{k+j}\left(q^{-r / 2} t ; q\right)\right),
\end{aligned}
$$

with

$$
\left[\begin{array}{l}
r \\
j
\end{array}\right]_{q}=\frac{(q ; q)_{r}}{(q ; q)_{j}(q ; q)_{r-j}}
$$

the q-binomial coefficient. 
Proof. On the one hand, using (??), we have that

$$
e_{q}\left(\frac{q^{r} t z}{2}\right) e_{q}\left(\frac{t z^{-1}}{2}\right)=\sum_{k=-\infty}^{\infty} I_{k}\left(q^{r / 2} t ; q\right) q^{\frac{k r}{2}} z^{k}
$$

and on the other hand, using (??) and (??), we find that

$$
\begin{aligned}
& e_{q}\left(\frac{q^{r} t z}{2}\right) e_{q}\left(\frac{t z^{-1}}{2}\right)=\left((1-q) \frac{t z}{2} ; q\right)_{r} e_{q}\left(\frac{t z}{2}\right) e_{q}\left(\frac{t z^{-1}}{2}\right) \\
= & \left(\sum_{j=0}^{r}\left[\begin{array}{l}
r \\
j
\end{array}\right]_{q} q^{\frac{j(j-1)}{2}}\left(\frac{q-1}{2}\right)^{j} t^{j} z^{j}\right)\left(\sum_{k=-\infty}^{\infty} I_{k}(t ; q) z^{k}\right),
\end{aligned}
$$

where in the last equality, we have used the $q$-binomial theorem (see [?], p. 20). Comparing the coefficients of $z^{k}$ in (??) and (??), and substituting $q^{-r / 2} t$ for $t$, we obtain that

$$
q^{\frac{k r}{2}} I_{k}(t ; q)=\sum_{j=0}^{r}\left[\begin{array}{l}
r \\
j
\end{array}\right]_{q} q^{\frac{j(j-r-1)}{2}}\left(\frac{q-1}{2}\right)^{j} t^{j} I_{k-j}\left(q^{-r / 2} t ; q\right) .
$$

A similar argument starting with $e_{q}\left(\frac{t z}{2}\right) e_{q}\left(\frac{q^{r} t z^{-1}}{2}\right)$, leads to

$$
q^{-\frac{k r}{2}} I_{k}(t ; q)=\sum_{j=0}^{r}\left[\begin{array}{l}
r \\
j
\end{array}\right]_{q} q^{\frac{j(j-r-1)}{2}}\left(\frac{q-1}{2}\right)^{j} t^{j} I_{k+j}\left(q^{-r / 2} t ; q\right) .
$$

Subtracting this equation from the previous one, leads to (??). This finishes the proof.

5. The Discrete heAT EQUation $D_{q, t} u=\left(L+\frac{(q-1) t}{4} I d\right) u$

In order to obtain a "finite number of terms phenomenon" for the fundamental solution of a discrete heat equation as in (??), when $L$ is a Toda $g$-soliton, we shall need to discretize the heat equation not only with respect to the space variable $x$, but also the time variable $t$.

The (partial) $q$-derivative $D_{q, t} f$ of a function $f(n, t), n \in \mathbb{Z}, t \in \mathbb{R}$, with respect to $t$, is given by

$$
\left(D_{q, t} f\right)(n, t)=\frac{f(n, q t)-f(n, t)}{(q-1) t} .
$$

From the definition (??) of the $q$-exponential, it is straightforward to check that

$$
D_{q, t} e_{q}(t)=e_{q}(t)
$$

In the next proposition, we deduce from the orthogonality relations (??) a formula for the fundamental solution of a (purely) discrete heat equation. 
Proposition 5.1. Let $L$ be a Toda g-soliton, as in Definition 3.1 and let $R=\min _{1 \leq j \leq g}\left|z_{j}\right|<1$. Given $m \in \mathbb{Z}$, the solution $u(n, t)$ of

$$
D_{q, t} u=\left(L+\frac{(q-1) t}{4} I d\right) u
$$

with initial condition

$$
u(n, 0)=\delta_{n m},
$$

is given by

$$
\begin{aligned}
K(m \mid n, t)=\frac{1}{2 \pi i} & \oint e_{q}\left(\frac{t z}{2}\right) e_{q}\left(\frac{t z^{-1}}{2}\right) \phi(n, z) \phi\left(m, z^{-1}\right) \frac{d z}{z} \\
& +\sum_{j=1}^{g} c_{j}^{2} e_{q}\left(\frac{t z_{j}}{2}\right) e_{q}\left(\frac{t z_{j}^{-1}}{2}\right) \phi\left(n, z_{j}\right) \phi\left(m, z_{j}\right),
\end{aligned}
$$

for $|t|<\frac{2 R}{1-q}$, with $\oint$ denoting the integral over the unit circle in the complex z plane.

Proof. As long as $|t|<2 /(1-q)$, the unit circle lies inside the annulus $(1-q)|t| / 2<|z|<2 /((1-q)|t|)$ for which the product $e_{q}\left(\frac{t z}{2}\right) e_{q}\left(\frac{t z^{-1}}{2}\right)$ is well defined. The stronger condition that $|t|<2 R /(1-q)$ with $R=\min _{1 \leq j \leq g}\left|z_{j}\right|$, guarantees that all the bound states are also within the same annulus. A simple computation, using (??) and (??), gives

$$
D_{q, t}\left(e_{q}\left(\frac{t z}{2}\right) e_{q}\left(\frac{t z^{-1}}{2}\right)\right)=\left(\frac{z+z^{-1}}{2}+\frac{(q-1) t}{4}\right) e_{q}\left(\frac{t z}{2}\right) e_{q}\left(\frac{t z^{-1}}{2}\right) \text {. }
$$

Using this equation and the fact that $\phi(n, z)$ solves the three-term recurrence relation (??), by taking $D_{q, t}$ of (??), we get

$$
\begin{aligned}
D_{q, t} K(m \mid n, t)=a_{n-1} K & (m \mid n-1, t)+b_{n} K(m \mid n, t) \\
& +a_{n} K(m \mid n+1, t)+\frac{(q-1) t}{4} K(m \mid n, t),
\end{aligned}
$$

which is (??). Putting $t=0$ in (??), by the orthogonality relations (??), we obtain (??). This establishes the proposition.

We now show that for an arbitrary Toda $g$-soliton, the fundamental solution (??) of (??) can be expanded as an infinite series in terms of the Jackson's $q$-Bessel functions of an imaginary argument $I_{k}(t ; q)$, that were introduced in (??). 
Theorem 5.2. Let $R=\min _{1 \leq j \leq g}\left|z_{j}\right|<1$. Then, for $|t|<\frac{2 R}{1-q}$, the fundamental solution $K(m \mid n, t)$ of (??) admits the following convergent series expansion

$$
\begin{aligned}
K(m \mid n, t) & =\sqrt{\frac{\tau_{m-1} \tau_{n}}{\tau_{m} \tau_{n-1}}} I_{m-n}(t ; q) \\
+ & \sum_{j=1}^{g} c_{j}^{2} \phi\left(m, z_{j}\right) \phi\left(n, z_{j}\right)\left\{\sum_{k=-\infty}^{m-n-1}\left(z_{j}^{k}-z_{j}^{-k}\right) I_{k}(t ; q)\right\} .
\end{aligned}
$$

Proof. The condition $|t|<2 R /(1-q)$ guarantees that the unit circle and the bound states lie inside the annulus of convergence $(1-q)|t| / 2<$ $|z|<2 /((1-q)|t|)$ of the Laurent expansion (??), which defines the functions $I_{k}(t ; q)$. Thus, substituting (??) into (??), the infinite series in the first term, being uniformly convergent on the unit circle, can pass through the integral, and the one appearing in the second term involving the bound states converges too, so that

$$
\begin{array}{r}
K(m \mid n, t)=\sum_{k=-\infty}^{\infty} I_{k}(t ; q)\left\{\frac{1}{2 \pi i} \oint z^{k-1} \phi(n, z) \phi\left(m, z^{-1}\right) \mathrm{d} z\right. \\
\left.+\sum_{j=1}^{g} c_{j}^{2} z_{j}^{k} \phi\left(n, z_{j}\right) \phi\left(m, z_{j}\right)\right\}
\end{array}
$$

We evaluate the integrals in (??) by residues, taking into account that the integrand has poles inside the unit circle only at $z=0$ and $z=$ $z_{1}, \ldots, z_{g}$. We compute

$$
\begin{aligned}
& \operatorname{res}_{z=z_{j}} z^{k-1} \phi(n, z) \phi\left(m, z^{-1}\right) \\
& =-\sqrt{\frac{\tau_{m}}{\tau_{m-1}}} \varepsilon_{j} z_{j}^{k-1} \phi\left(n, z_{j}\right) w^{*}\left(m, 0, z_{j}^{-1}\right), \operatorname{using}(? ?),(? ?),(? ?), \\
& =-c_{j}^{2} z_{j}^{k} \phi\left(n, z_{j}\right) \phi\left(m, z_{j}\right) \text {, using (??),(??),(??). }
\end{aligned}
$$

This shows that (??) reduces to

$$
K(m \mid n, t)=\sum_{k=-\infty}^{\infty} I_{k}(t ; q) \operatorname{res}_{z=0} z^{k-1} \phi(n, z) \phi\left(m, z^{-1}\right) .
$$

From (??) and (??), we have

$$
z^{k-1} \phi(n, z) \phi\left(m, z^{-1}\right)=\sqrt{\frac{\tau_{n-1} \tau_{m}}{\tau_{n} \tau_{m-1}}} z^{k} w(n-1,0, z) w^{*}(m, 0, z) \text {. }
$$


Expanding the right-hand side of (??) near $z=0$, using (??), we find that

$$
z^{k-1} \phi(n, z) \phi\left(m, z^{-1}\right)=z^{k+n-m-1}\left(\sqrt{\frac{\tau_{n} \tau_{m-1}}{\tau_{n-1} \tau_{m}}}+O(z)\right) \text {, near } z=0,
$$

which gives

$$
\operatorname{res}_{z=0} z^{k-1} \phi(n, z) \phi\left(m, z^{-1}\right)=\left\{\begin{array}{l}
0 \quad \text { if } k \geq m-n+1, \\
\sqrt{\frac{\tau_{n} \tau_{m-1}}{\tau_{n-1} \tau_{m}}} \text { if } k=m-n .
\end{array}\right.
$$

From (??), we deduce that

$$
\begin{aligned}
& \operatorname{res}_{z=\infty} z^{k} w(n-1,0, z) w^{*}(m, 0, z) \\
& =-\frac{\tau_{n} \tau_{m-1}}{\tau_{n-1} \tau_{m}} \operatorname{res}_{z=0} z^{-k} w^{*}(n, 0, z) w(m-1,0, z), \\
& =0 \quad \text { when } k \leq m-n-1,
\end{aligned}
$$

where the last equality follows again from (??). By the residue theorem on the Riemann sphere, we conclude that

$$
\begin{aligned}
& \operatorname{res}_{z=0} z^{k-1} \phi(n, z) \phi\left(m, z^{-1}\right)= \\
& -\sum_{j=1}^{g}\left\{\operatorname{res}_{z=z_{j}}+\operatorname{res}_{z=z_{j}^{-1}}\right\} z^{k-1} \phi(n, z) \phi\left(m, z^{-1}\right), \quad \text { when } k \leq m-n-1 .
\end{aligned}
$$

It remains to evaluate the residues appearing on the right-hand side of (??). We have

$$
\begin{aligned}
& -\left\{\operatorname{res}_{z=z_{j}}+\operatorname{res}_{z=z_{j}^{-1}}\right\} z^{k-1} \phi(n, z) \phi\left(m, z^{-1}\right) \\
= & \varepsilon_{j} \sqrt{\frac{\tau_{n-1} \tau_{m}}{\tau_{n} \tau_{m-1}}} w\left(n-1,0, z_{j}\right) w^{*}\left(m, 0, z_{j}^{-1}\right)\left(z_{j}^{k}-z_{j}^{-k}\right) \\
& \operatorname{using}(? ?),(? ?) \text { and }(? ?), \\
= & c_{j}^{2} \phi\left(n, z_{j}\right) \phi\left(m, z_{j}\right)\left(z_{j}^{k}-z_{j}^{-k}\right) \\
& \operatorname{using}(? ?),(? ?) \text { and }(? ?) .
\end{aligned}
$$

Combining (??), (??), (??) and (??) leads to the announced result (??). This concludes the proof of the theorem.

Remark 5.3. If we replace in (??) the $q$-exponential by the usual exponential as in (??), we obtain the fundamental solution of the "timecontinuous, space-discrete" heat equation (??). Then, the same argument as in Theorem 5.2 shows that (??) admits a series expansion as in (??), with $I_{k}(t ; q)$ replaced by the standard Bessel functions $I_{k}(t)$ 
of imaginary argument, which converges now for all $t$. However, the standard identities satisfied by the functions $I_{k}(t)$ will never reduce this infinite series to a finite sum. This only happens in the limiting case considered in [?], when all the bound states $z_{j}$ accumulate at \pm 1 .

Our final result shows that the introduction of the $q$-exponential in (??) makes it possible to find some special choices of the bound states $z_{j}$, for which the infinite expansion (??) reduces to a finite sum.

Corollary 5.4. Let $0<q<1$. Let $L$ be a Toda g-soliton as in Definition 3.1, with discrete spectrum at

$$
z_{1}= \pm q^{\frac{r_{1}}{2}}, \ldots, z_{g}= \pm q^{\frac{r_{g}}{2}}
$$

for some choice of positive integers $r_{1}, \ldots, r_{g}$ and some arbitrary choice of the \pm signs, in such a way that the $z_{j}$ 's are distinct points. Then, for $|t|<\frac{2 R}{1-q}$ with $R$ as in Theorem 5.2, the expansion (??) reduces to a finite sum of the form

$$
\begin{aligned}
& K(m \mid n, t)=\sqrt{\frac{\tau_{m-1} \tau_{n}}{\tau_{m} \tau_{n-1}}} I_{m-n}(t ; q) \\
& \quad+\sum_{j=1}^{g} \sum_{k=m-n-r_{j}}^{m-n+r_{j}-1} p_{j, k}(m \mid n, t) I_{k}\left(q^{-r_{j} / 2} t ; q\right),
\end{aligned}
$$

with $p_{j, k}(m \mid n, t)$ polynomials in $t$ of degree $r_{j}$, that can be computed explicitly.

Proof. In view of (??), it suffices to show that when $z=\epsilon q^{r / 2}, r=$ $1,2, \ldots, \epsilon= \pm 1$, the series

$$
\sum_{k=-\infty}^{m-n-1}\left(z^{k}-z^{-k}\right) I_{k}(t ; q)
$$

can be expressed as a finite sum. But this fact follows immediately from the property (??) that we established in Proposition 4.1, which shows that in these cases the series above reduces to a telescoping one. Explicitly, we find that

$$
\begin{aligned}
\sum_{k=-\infty}^{m-n-1} \epsilon^{k}\left(q^{\frac{k r}{2}}-q^{-\frac{k r}{2}}\right) I_{k}(t ; q)= & \\
-\epsilon^{m-n-1} \sum_{k=0}^{r-1} & \left(\sum_{j=k+1}^{r} \epsilon^{j+k}\left[\begin{array}{l}
r \\
j
\end{array}\right]_{q} q^{\frac{j(j-r-1)}{2}}\left(\frac{q-1}{2}\right)^{j} t^{j}\right) \\
& \times\left(I_{m-n-k-1}\left(q^{-r / 2} t ; q\right)+\epsilon I_{m-n+k}\left(q^{-r / 2} t ; q\right)\right) .
\end{aligned}
$$


Only the functions $I_{k}\left(q^{-r / 2} t ; q\right), m-n-r \leq k \leq m-n+r-1$, are involved in this sum, and their coefficients are polynomials in $t$ of degree $r$. This concludes the proof of the corollary.

In $[?, ?, ?]$, the following difference - $q$-difference bispectral problem was studied. To find all doubly infinite (or semi-infinite) Jacobi matrices for which some family of eigenfunctions is also a family of eigenfunctions of a $q$-difference operator of an arbitrary order, in the spectral variable $z$. The Askey-Wilson orthogonal polynomials [?] provide instances of semi-infinite Jacobi matrices which solve this problem. Rank 1 solutions of the problem, that we called "Askey-Wilson type solitons", were described in [?] (see also [?], which further contains a description of rank 2 solutions). It is quite remarkable that the conditions (??) which lead to a "finite sum formula" for the fundamental solution of (??), precisely define the "Askey-Wilson type solitons". It would be interesting to have an explanation for this coincidence!

Finally, our results should be extended to the heat equation (??), with $V(x)$ a soliton of the KdV equation. Clearly, the circle in (??) should be replaced by the line. The limiting case when all the bound states accumulate at the origin, should be studied too, leading hopefully to a proof of the results conjectured in [?] about the rational solutions of the $\mathrm{KdV}$ equation. I plan to return to these questions in a further publication.

Acknowledgments. The support of a Research Grant (2001-2004) of the Belgian National Fund for Scientific Research is gratefully acknowledged.

\section{REFERENCES}

[1] R. Askey and J. Wilson, Some basic hypergeometric orthogonal polynomials that generalize Jacobi polynomials, Mem. Amer. Math. Soc. No. 319, 1985.

[2] Yu. Berest, "Huygens' principle and the bispectral problem", in: The Bispectral Problem, J. Harnad and A. Kasman (eds.), CRM Proc. Lecture Notes 14, Amer. Math. Soc., Providence, RI, 1998, pp. 11-30.

[3] Yu. Berest and A. P. Veselov, Hadamard's problem and Coxeter groups: new examples of Huygens' equations, Funktsional. Anal. i Prilozhen. 28 (1) (1994), 3-15 (Russian); English transl., Funct. Anal. Appl. 28 (1) (1994), 3-12.

[4] Ju. M. Berezanskii, Expansions in Eigenfunctions of Selfadjoint Operators, Transl. Math. Monographs 17, Amer. Math. Soc., Providence, 1968.

[5] P. Deift, L. C. Li and C. Tomei, Toda flows with infinitely many variables, J. Funct. Anal. 64 (1985), 358-402.

[6] P. Deift and E. Trubowitz, Inverse scattering on the line, Comm. Pure Appl. Math. 32 (1979), 121-251. 
[7] J. F. van Diejen and A. N. Kirillov, Formulas for q-spherical functions using inverse scattering theory of reflectionless Jacobi operators, Commun. Math. Phys. 210 (2000), 335-369.

[8] J. J. Duistermaat and F. A. Grünbaum, Differential equations in the spectral parameter, Commun. Math. Phys. 103 (1986), 177-240.

[9] H. Flaschka, On the Toda lattice II - Inverse scattering solution, Progr. Theor. Phys. 51 (3) (1974), 703-716.

[10] G. Gasper and M. Rahman, Basic hypergeometric series, Encyclopedia of Mathematics and Its Applications 35, Cambridge University Press (1990).

[11] F. A. Grünbaum, "Some bispectral musings", in: The Bispectral Problem, J. Harnad and A. Kasman (eds.), CRM Proc. Lecture Notes 14, Amer. Math. Soc., Providence, RI, 1998, pp. 31-45.

[12] F. A. Grünbaum, "The bispectral problem: an overview", in: Special Functions 2000: Current Perspective and Future Directions, J. Bustoz, M. E. H. Ismail and S. K. Suslov (eds.), NATO Adv. Sci. Inst. Ser. C Math. Phys. Sci., Kluwer, 2001, pp. 129-140.

[13] F. A. Grünbaum and L. Haine, Some functions that generalize the AskeyWilson polynomials, Commun. Math. Phys. 184 (1997), 173-202.

[14] F. A. Grünbaum and L. Haine, Associated polynomials, spectral matrices and the bispectral problem, Meth. Appl. Anal. 6 (2) (1999), 209-224.

[15] F. A. Grünbaum and P. Iliev, Heat kernel expansions on the integers, Math. Phys. Anal. Geom. 5 (2002), 183-200.

[16] L. Haine, "The q-hypergeometric equation, Askey-Wilson type solitons and rational curves with singularities", in: The Kowalevski Property, V. B. Kuznetsov (ed.), CRM Proc. Lecture Notes 32, Amer. Math. Soc., Providence, RI, 2002, pp. 69-91.

[17] L. Haine and P. Iliev, Commutative rings of difference operators and an adelic flag manifold, Internat. Math. Res. Notices 6 (2000), 281-323.

[18] L. Haine and P. Iliev, A rational analogue of the Krall polynomials, J. Phys. A: Math. Gen. 34 (2001), 2445-2457.

[19] L. Haine and P. Iliev, Askey-Wilson type functions, with bound states, The Ramanujan Journal (in press).

[20] M. E. H. Ismail, The basic Bessel functions and polynomials, SIAM J. Math. Anal. 12 (1981), 454-468.

[21] F. H. Jackson, The application of basic numbers to Bessel's and Legendre's functions, Proc. London Math. Soc. (2) 2 (1905), 192-220.

[22] M. Kac, Integration in Function Spaces and Some of Its Applications, Lezioni Fermiane, Pisa, 1980.

[23] I. M. Krichever, Algebraic curves and nonlinear difference equations Uspekhi Mat. Nauk 33 (1978), 215-216; English transl. in Russian Math. Surveys 33 (1978), 255-256.

[24] H. P. McKean and P. van Moerbeke, The spectrum of Hill's equation, Inventiones Math. 30 (1975), 217-274.

[25] P. van Moerbeke and D. Mumford, The spectrum of difference operators and algebraic curves, Acta Math. 143 (1979), 93-154.

[26] F. W. Nijhoff and O. A. Chalykh, Bispectral rings of difference operators, Russian Math. Surveys 54 (1999), 644-645. 
[27] R. Schimming, An explicit expression for the Korteweg-de Vries hierarchy, Z. Anal. Anwendungen 7 (1988), 203-214.

[28] G. Segal and G. Wilson, Loop groups and equations of KdV type, Inst. Hautes Études Sci. Publ. Math. 61 (1985), 5-65.

[29] M. Toda, Theory of Nonlinear Lattices, Springer Series in Solid-State Sciences 20, Springer, Berlin, Heidelberg, New-York, 1981.

[30] G. Wilson, Bispectral commutative ordinary differential operators, J. Reine Angew. Math. 442 (1993), 177-204.

Department of Mathematics, Université Catholique de Louvain, Chemin du Cyclotron 2, 1348 Louvain-la-Neuve, Belgium

E-mail address: haine@math.ucl.ac.be 